University of Nebraska - Lincoln

DigitalCommons@University of Nebraska - Lincoln

U.S. Department of Veterans Affairs Staff

Publications

U.S. Department of Veterans Affairs

2006

Development and evaluation of a multifaceted ergonomics program to prevent injuries associated with patient handling tasks

Audrey Nelson

James A. Haley Veterans Hospital, audrey.nelson@med.va.gov

Mary Matz

James A. Haley Veterans Hospital, Mary.Matz@med.va.gov

Fangfei Chen

James A. Haley Veterans Hospital, fangfei.chen@med.va.gov

Kris Siddharthan

James A. Haley Veterans Hospital, Kris.siddharthan@med.va.gov

John Lloyd

James A. Haley Veterans Hospital, john.lloyd@med.va.gov

See next page for additional authors

Follow this and additional works at: https://digitalcommons.unl.edu/veterans

Nelson, Audrey; Matz, Mary; Chen, Fangfei; Siddharthan, Kris; Lloyd, John; and Fragala, Guy, "Development and evaluation of a multifaceted ergonomics program to prevent injuries associated with patient handling tasks" (2006). U.S. Department of Veterans Affairs Staff Publications. 59.

https://digitalcommons.unl.edu/veterans/59

This Article is brought to you for free and open access by the U.S. Department of Veterans Affairs at DigitalCommons@University of Nebraska - Lincoln. It has been accepted for inclusion in U.S. Department of Veterans Affairs Staff Publications by an authorized administrator of DigitalCommons@University of Nebraska - Lincoln. 


\section{Authors}

Audrey Nelson, Mary Matz, Fangfei Chen, Kris Siddharthan, John Lloyd, and Guy Fragala 


\title{
Development and evaluation of a multifaceted ergonomics program to prevent injuries associated with patient handling tasks
}

\author{
Audrey Nelson ${ }^{\mathrm{a}, \mathrm{b}, *}$, Mary Matz ${ }^{\mathrm{a}}$, Fangfei Chen $^{\mathrm{a}}$, Kris Siddharthan ${ }^{\mathrm{a}}$, \\ John Lloyd ${ }^{\mathrm{a}, \mathrm{c}}$, Guy Fragala ${ }^{\mathrm{d}}$ \\ ${ }^{a}$ Patient Safety Center of Inquiry, James A. Haley Veterans Hospital, 11605 N. Nebraska Avenue, Tampa, FL 33612,USA \\ ${ }^{\mathrm{b}}$ University of South Florida, College of Nursing, Tampa, FL, USA \\ ${ }^{\mathrm{c}}$ University of South Florida, College of Engineering, Tampa, FL, USA \\ ${ }^{\mathrm{d}}$ Environmental Health \& Engineering, Inc., Newton, MA,USA
}

Received 15 March 2005; received in revised form 1 August 2005; accepted 10 September 2005

\begin{abstract}
Problem statement: Nurses have one of the highest rates of work-related musculoskeletal injury of any profession. Over the past 30 years, efforts to reduce work-related musculoskeletal disorders in nurses have been largely unsuccessful.

Specific aims: The primary goal of this program was to create safer working environments for nursing staff who provide direct patient care. Our first objective was to design and implement a multifaceted program that successfully integrated evidence-based practice, technology, and safety improvement. The second objective was to evaluate the impact of the program on injury rate, lost and modified work days, job satisfaction, self-reported unsafe patient handling acts, level of support for program, staff and patient acceptance, program effectiveness, costs, and return on investment.

Intervention: The intervention included six program elements: (1) Ergonomic Assessment Protocol, (2) Patient Handling Assessment Criteria and Decision Algorithms, (3) Peer Leader role, "Back Injury Resource Nurses", (4) State-of-the-art Equipment, (5) After Action Reviews, and (6) No Lift Policy.

Methods: A pre-/post design without a control group was used to evaluate the effectiveness of a patient care ergonomics program on 23 high risk units (19 nursing home care units and 4 spinal cord injury units) in 7 facilities. Injury rates, lost work days, modified work days, job satisfaction, staff, and patient acceptance, program effectiveness, and program costs/savings were compared over two nine month periods: pre-intervention (May 2001-January 2002) and post-intervention (March 2002-November 2002). Data were collected prospectively through surveys, weekly process logs, injury logs, and cost logs.

Results: The program elements resulted in a statistically significant decrease in the rate of musculoskeletal injuries as well as the number of modified duty days taken per injury. While the total number of lost workdays decreased by $18 \%$ post-intervention, this difference was not statistically significant. There were statistically significant increases in two subscales of job satisfaction: professional status and tasks requirements. Self-reports by nursing staff revealed a

\footnotetext{
The study was supported by the Department of Veterans Affairs, Veterans Health Administration, New Program Initiatives \#00019-1.

*Corresponding author. Tel.: + 18135583902 ; fax: + 18135583991.

E-mail addresses: valerie.kelleher@med.va.gov, audrey.nelson@med.va.gov (A. Nelson).
} 
statistically significant decrease in the number of 'unsafe' patient handling practices performed daily. Nurses ranked program elements they deemed to be "extremely effective": equipment was rated as most effective ( $96 \%$ ), followed by No Lift Policy $(68 \%)$, peer leader education program (66\%), ergonomic assessment protocol (59\%), patient handling assessment criteria and decision algorithms (55\%), and lastly after action reviews (41\%). Perceived support and interest for the program started at a high level for managers and nursing staff and remained very high throughout the program implementation. Patient acceptance was moderate when the program started but increased to very high by the end of the program. Although the ease and success of program implementation initially varied between and within the facilities, after six months there was strong evidence of support at all levels. The initial capital investment for patient handling equipment was recovered in approximately 3.75 years based on annual post-intervention savings of over $\$ 200,000 /$ year in workers' compensation expenses and cost savings associated with reduced lost and modified work days and worker compensation.

Conclusions: This multi-faceted program resulted in an overall lower injury rate, fewer modified duty days taken per injury, and significant cost savings. The program was well accepted by patients, nursing staff, and administrators. Given the significant increases in two job satisfaction subscales (professional status and task requirements), it is possible that nurse recruitment and retention could be positively impacted.

(C) 2005 Elsevier Ltd. All rights reserved.

Keywords: Safe patient handling and movement; Patient safety; Ergonomics; Nursing; Nursing injuries

What is already known about the topic?:

- Nursing is a high risk occupation, and performance of patient handling tasks contributes significantly to this occupational risk.

- Interventions to reduce risk associated with patient handling tasks have been based on tradition and personal experience rather than scientific evidence.

- Patient handling is most commonly performed manually, augmented with classes in body mechanics and training in lifting techniques. Despite the fact that these strategies are NOT evidence-based, and have been deemed ineffective, they are still widely used and taught in schools of nursing.

- There is moderate evidence that multifactor interventions based on risk assessment are more successful than an individual intervention.

\section{What this paper adds?:}

- This program resulted in improvements in injury rates, modified duty days, job satisfaction, costs, and self-reported performance of 'unsafe' patient handling practices. The number of lost work days decreased, but was not statistically significant.

- Nurses ranked program elements in order of their perceived impact: equipment, no lift policy, peer leader education program, ergonomic assessment protocol, patient handling assessment criteria and decision algorithms, and lastly after action reviews.

- There was strong support for the program from caregivers, patients, and administrators.

- The return on investment was recovered in approximately 3.75 years.

\section{Problem statement}

Nurses have one of the highexst incidences of work related back injuries of any profession (Bureau of Labor statistics BLS, 2002), estimated at 12.6/100 full time workers (Bureau of Labor Statistics [BLS], 2003). This number is considered to be a low estimate, since underreporting of injuries in nursing is common (US Department of Health and Human Services (USDHHS), 1999). Data from over 80 studies across a number of countries indicated that back injury to nurses have a worldwide point prevalence of approximately 17 percent, an annual prevalence of 40-50 percent and a lifetime prevalence of 35-80 percent (Hignett, 1996). There has been a steady decline in occupational injuries starting in 1992, but when work-related injuries for patient care providers are examined, no such improvement is noted (Fragala and Bailey, 2003).

Over the past 30 years, most efforts to reduce workrelated musculoskeletal disorders in nursing have focused on body mechanics and lifting techniques. However, these efforts have consistently failed to reduce the risk associated with patient handling and movement (Anderson, 1980; Brown, 1972; Buckle, 1981, 1987; Daltroy, 1997; Daws, 1981; Dehlin et al., 1976; Harber et al., 1994; Hayne, 1984; Lagerstrom and Hagberg, 1997; Larese and Fiorito, 1994; Shaw, 1981; Snook et al., 1978; St. Vincent et al., 1989; Stubbs et al., 1983; Venning, 1988). In 2002, the Veterans Health Administration spent approximately $\$ 22$ million over a oneyear period in costs associated with injuries related to patient handling (Hodgson, 2001). Preventative interventions are critically needed to control the hazards and economic burdens associated with patient handling tasks. With the existing nursing shortage, efforts to improve safety and decrease work-related injuries could 
have a huge impact on the recruitment and retention of qualified nursing staff.

\section{Background}

Many patient handling tasks are considered to be high-risk, based on the magnitude of weight lifted, awkwardness and unpredictable nature of the load lifted (patient), and sustained awkward positions used to provide nursing care. Historically, patient handling injury-reduction strategies have been based on tradition and personal experience rather than scientific evidence. The most commonly approach is manual patient lifting, augmented with classes training in body mechanics, training in safe lifting techniques, and use of back belts. There is strong evidence that these approaches are NOT effective in reducing caregiver injuries (Nelson et al., 2003a; Nelson and Baptiste, 2004; in press). Promising new interventions are now being tested. Given the complexity of this high-risk, high volume, high-cost problem, multifaceted programs are more likely to be effective than any single intervention (Panel on Musculoskeletal Disorders in the Workplace, 2001; Stetler et al., 2003).

Hignett (2003) summarized 63 studies and graded the evidence related to patient handling interventions. She grouped the studies into three categories: multifactor interventions, single factor interventions, and training interventions; she concluded:

- There is moderate evidence that multifactor interventions based on risk assessment are successful.

- There is moderate evidence that multifactor interventions NOT based on risk assessment are successful.

- There is moderate evidence that a single factor intervention (patient handling equipment alone) is successful.

- There is moderate evidence that a single factor intervention, lift teams are successful.

- There is strong evidence that technique training interventions are not effective in improving work practices or reducing injury rates.

- There is moderate evidence that technique training interventions have mixed results in the short term.

In 1994, Lahad et al. (1994) reviewed evidence for four interventions to prevent low back pain. Studies published between 1966 and 1993 were included $(n=64)$. There is limited evidence that exercises to strengthen back or abdominal muscles and improve fitness will decrease incidence and duration of low back pain episodes. There is minimal support for education strategies and insufficient evidence to recommend back belts. There were no published studies that examined risk factor modification (smoking cessation and weight loss), but it has merit for future research.

In 2003, Stetler et al. summarized literature from the 1990s (Stetler et al., 2003) to develop a safe patient handling plan for a hospital. The plan included external evidence (research findings) and internal evidence (systematic data collected on site to refine and improve program). They determined:

- No simple solution or single intervention would be effective.

- Multifaceted interventions should include at least two of the following: elimination of risk factors, engineering controls, administrative controls (no lift policy), training/education.

- Successful evidence-based strategies for changing behavior include: opinion leaders, educational outreach, reminder systems, and audit/feedback.

- Given the scope of this problem, further research is needed to test multifaceted programs to create safer work environments for caregivers. Such was the goal of this study.

\section{Goals and objectives}

The primary goal of this program was to create safer working environments for nursing staff who provide direct patient care. Our first objective was to design and implement a multifaceted program that successfully integrated evidence-based practice, technology, and safety improvement. The second objective was to evaluate the impact of the program on injury rate, lost and modified work days, job satisfaction, self-reported unsafe patient handling acts, level of support for program, staff and patient acceptance, program effectiveness, costs, and return on investment.

\section{Program description}

A multifaceted program with six program elements was designed after examining international case studies within and outside the healthcare industry (Nelson, 2003; Nelson et al., 2003b). The program elements included: (1) ergonomic assessment protocol, (2) patient handling assessment criteria and decision algorithms, (3) peer safety leaders, known as a Back Injury Resource Nurses (BIRNS), (4) patient handling equipment based on needs identified in the ergonomic assessment, (5) after action review (AAR) process, and (6) no lift policy. Details about these interventions have been published elsewhere (Nelson et al., 2003b) and can also be found on the web (www.visn8.med.va.gov/patientsafetycenter/). 


\section{Methods}

A pre/post design without a control group was used to evaluate the effectiveness of a patient care ergonomic program in 23 high risk units. Injury rates, lost and modified work days, job satisfaction, self-reported unsafe patient handling acts, level of support for program, staff and patient acceptance, program effectiveness, and costs were compared for the nine month pre-intervention (May 2001-January 2002) and the nine month post-intervention (February 2002-October 2002) periods. Data were collected prospectively through surveys, weekly process logs, injury logs, cost logs, and focus groups.

Study sites: Twenty-three (23) high-risk units (19 nursing home care units (NHCU) and four spinal cord injury (SCI) units) in seven facilities in southeast United States were included as research sites for implementation of this project. High-risk units were defined as inpatient hospital units with a high proportion of dependent patients, requiring full assistance with patient handling tasks, including lifting and activities of daily living. Historically, these units have the highest incidence and severity of job-related musculoskeletal injuries and are most likely to benefit from an effective program intervention. The 19 NHCUs ranged from 38 to 60 beds and were staffed with 19-53 nursing personnel. The 4 SCI units ranged from 20 to 56 beds and were staffed with 27-47 personnel. The baseline for these units varied, but most units had training programs and some equipment in place. One unit had ceiling mounted patient lifts in every room.

Measurement: Key measures included injury rate, lost and modified work days, job satisfaction, self-reported unsafe patient handling acts, level of support for program, staff and patient acceptance, program effectiveness, costs, and return on investment. Table 1 outlines the operational definition, data source, frequency of data collection, as well as the plan for analysis.

Sample: In the 23 participating units, there were a total of 1,133 nursing staff, of which $825(73 \%)$ signed an informed consent agreeing to participate. The sample included all direct patient care nursing staff $(n=825)$, including registered nurses (RN), licensed practical nurses (LPN), nursing aides (NA), student nurses, health care technicians assigned to perform patient care (GU tech, patient transport tech, shower team members), and nurse managers who performed direct patient care. Of all staff in the 23 units, a total of 462 staff were not characterized by their nurse job titles to enhance anonymity. Among the 671 staff with specified job titles, 216 (32\%) were NAs, $172(26 \%)$ were LPNs, $241(36 \%)$ were RNs, and $42(6 \%)$ were classified as 'Other'. A chi-square test $(p=0.58)$ determined there was no statistically significant differences in the dis- tribution of nurse job title among the consent and nonconsent staff. This sample was used for injury and cost data. The inclusion and exclusion criteria, as well as sample size differed for other data collection strategies, as described below.

Sample for surveys: A random sample $(n=300)$ of the 825 participating direct care nursing staff were invited to complete the surveys. An overall response rate of $70 \%$ $(N=209)$ was achieved for the pre-intervention survey. Post-intervention resignations, retirements, or transfers resulted in $30 \% \quad(n=90)$ loss to follow-up. $70 \%$ $(N=210)$ of the original 300 employees were still employed for the post-intervention survey. Of the 210 surveys distributed, the overall response rate was $79 \%$ $(N=166)$. There was little variation in response rate by job title or type of unit (SCI or NHCU).

Sample for focus groups: Direct care nursing staff on each high-risk unit were invited to participate in focus groups. A total of 18 staff focus groups were held, with 4-15 participants in each group. Nine focus groups were held at both the start and at the end of the program. The second round of focus groups included one management focus group at all but one site. Management personnel invited to participate included nurse managers, nurse administrators, nurse educators, clinical nurse specialists, safety staff, occupational health staff, and other management staff involved in the implementation of the program.

Case definition for injuries: Since the interventions were designed to prevent or minimize injuries related to patient handling, it is critical to exclude other injuries associated with keyboard tasks, falls in parking lot, etc. which are likely included in injury databases. To minimize this confounder, we developed an algorithm (case definition) to operationalized patient handling injuries. See Fig. 1. Only musculoskeletal injuries that occurred during patient handling tasks were included.

Data collection protocol: Data were collected prospectively through surveys, weekly process logs, injury logs, and cost logs. Data collection was coordinated at each site by a trained site coordinator. Subject participation included completion of surveys and providing the site coordinators with injury data if a case was identified. Subjects who reported a musculoskeletal injury that occurred during patient handling tasks were initially interviewed by the Site Coordinator and had monthly follow up interviews to track days away from work and injury-related costs. All data was handled as intent to treat and every effort was made to contact the subjects each month. The initial injury interview took approximately $20 \mathrm{~min}$ and the follow-up interview, on average, took less than $10 \mathrm{~min}$ given no significant follow-up information was given and up to $30 \mathrm{~min}$ if significant changes in the injury information occurred. Both injury data and $\log$ data were manually collected on word documents and entered by hand into an Access 


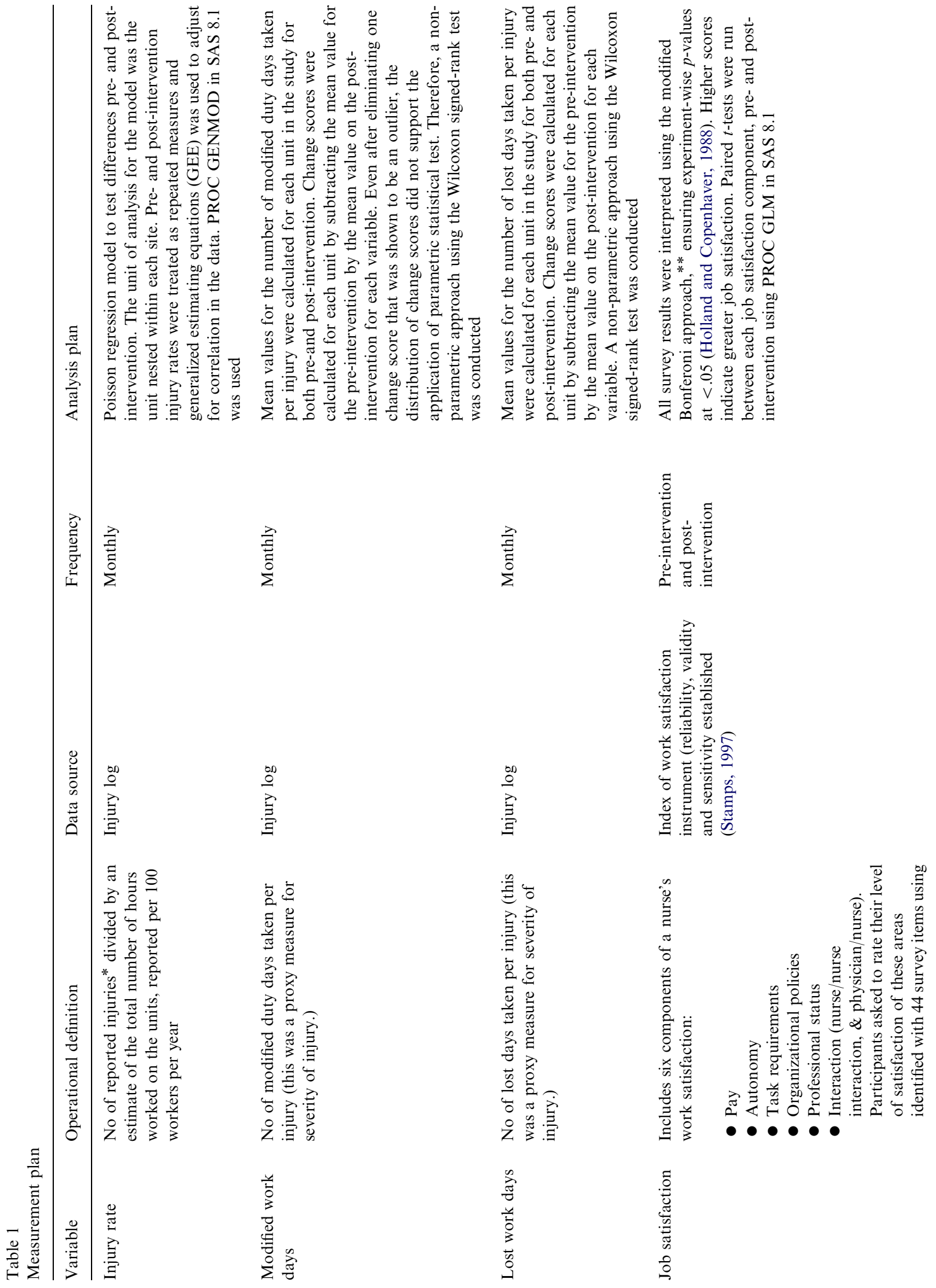




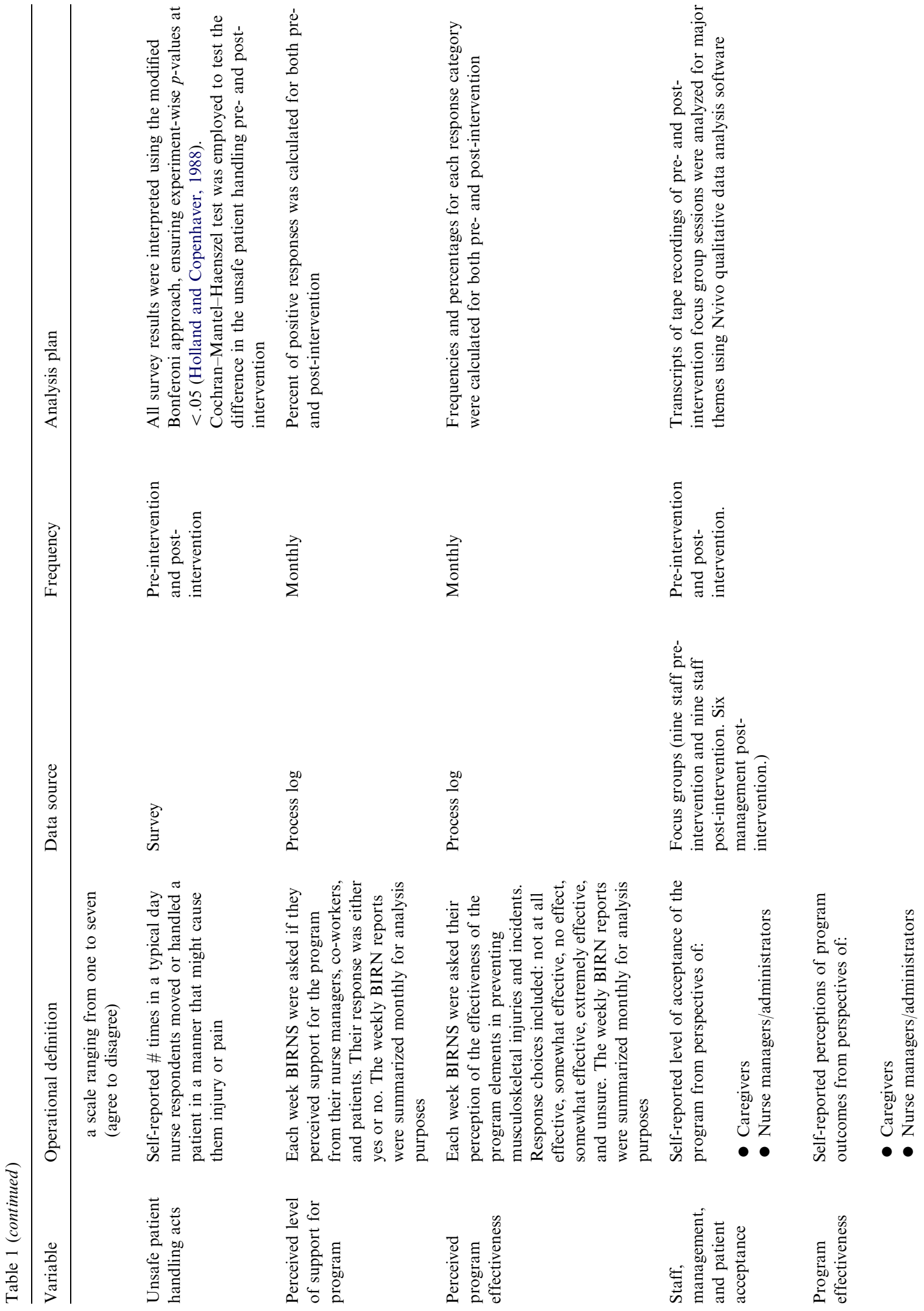



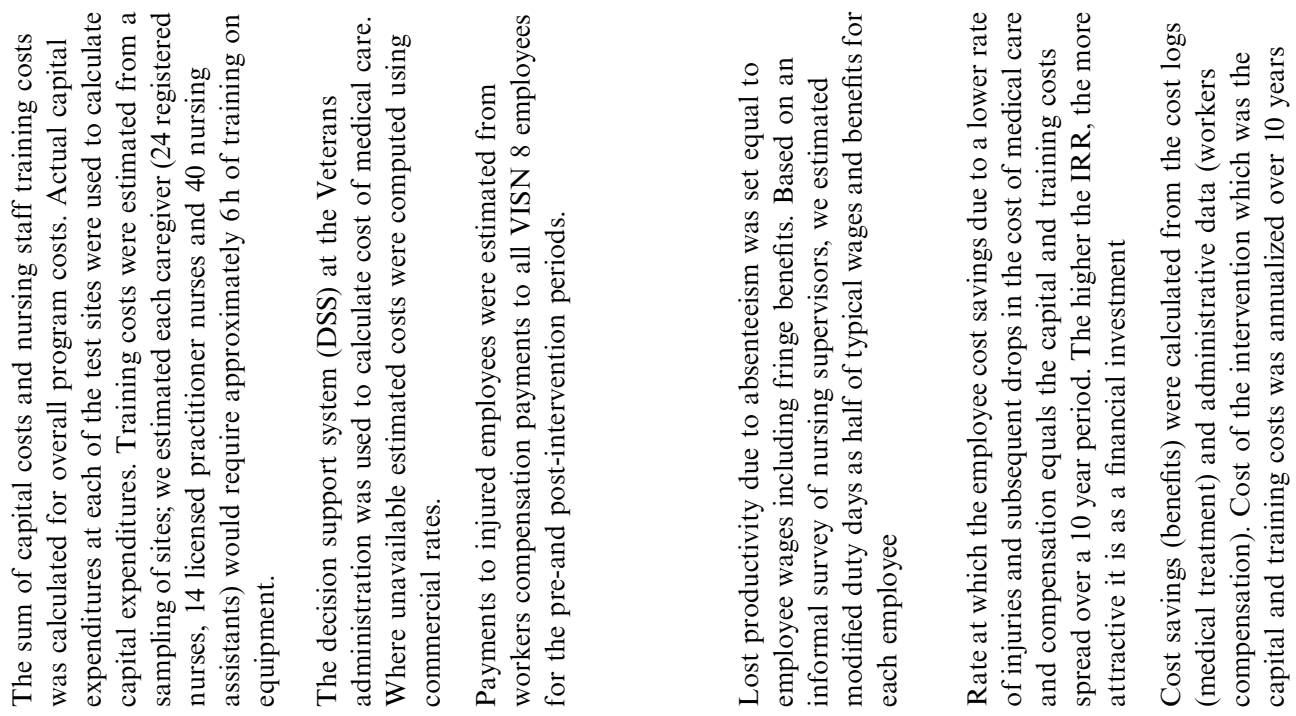

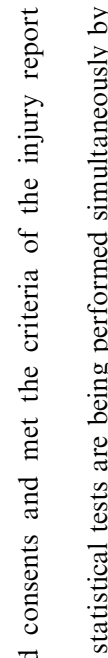

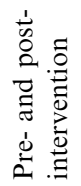
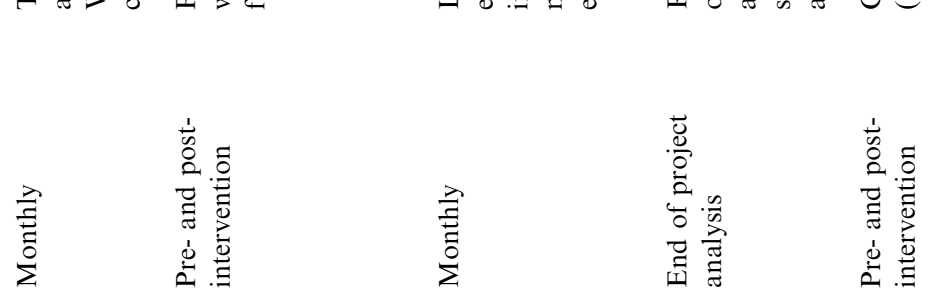

蒈
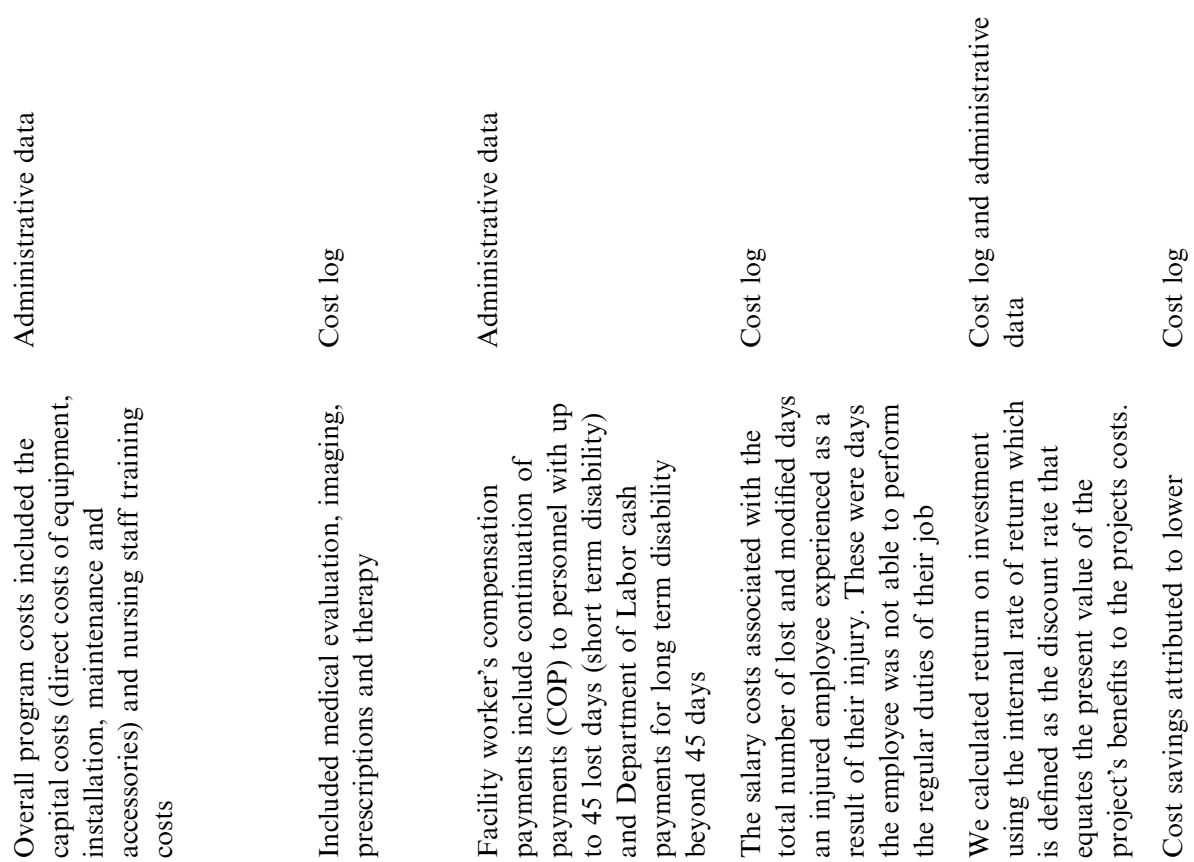

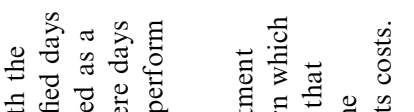

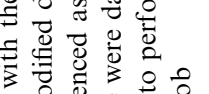

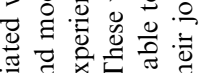

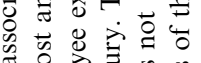

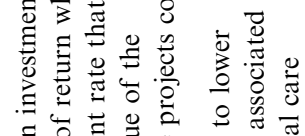

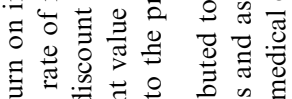

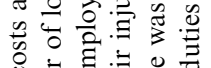

至

ठ

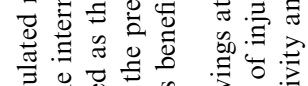

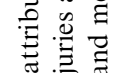

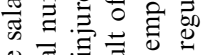

चु

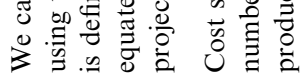

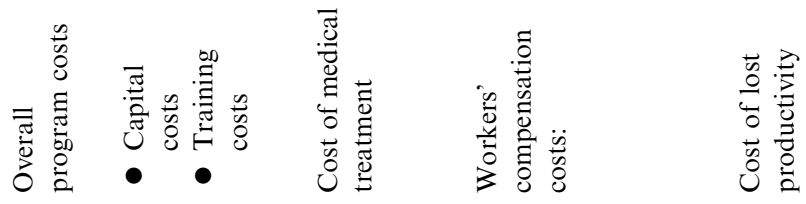

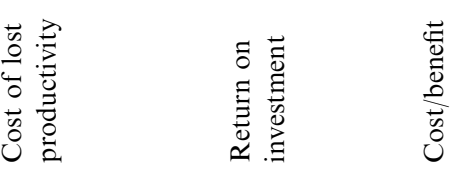

$\overrightarrow{0}$
0
0
0
0
0 


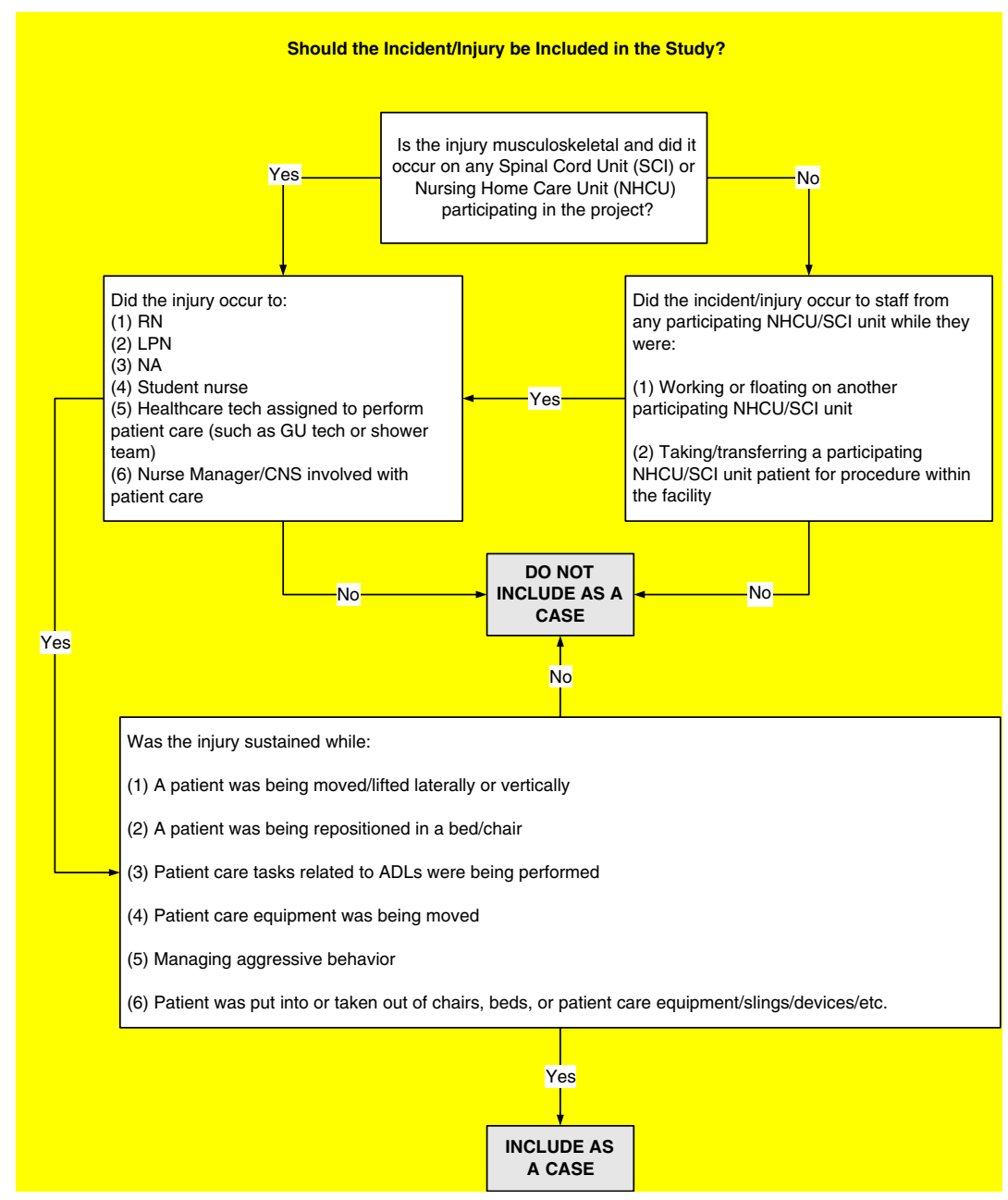

Fig. 1. Injury report algorithm.

database. A random sample of $20 \%$ were doublechecked for consistency.

Data analysis plan: A Poisson regression model was used to test injury rate differences pre- and postintervention. The unit of analysis for the model was the unit nested within each site. Pre- and post-intervention injury rates were treated as repeated measures and generalized estimating equations (GEE) were used to adjust for correlation in the data. PROC GENMOD in SAS 8.1 was used.

Mean values for the number of modified duty days taken per injury were calculated for each unit in the study for both pre- and post-intervention. Change scores were calculated for each unit by subtracting the mean value for the pre-intervention by the mean value on the post-intervention for each variable. Even after eliminating one change score that was shown to be an outlier, the distribution of change scores did not support the application of parametric statistical test. Therefore, a non-parametric approach using the Wilcoxon signedrank test was conducted. Mean values for the number of lost days taken per injury were calculated for each unit in the study for both pre- and post-intervention. Change scores were calculated for each unit by subtracting the mean value for the pre-intervention by the mean value on the post-intervention for each variable. A nonparametric approach using the Wilcoxon signed-rank test was conducted.

To test the change in job satisfaction, paired $t$-test was run between each job satisfaction component pre- and post-intervention using PROC GLM in SAS 8.1. All survey results were interpreted using the modified Bonferoni approach, ensuring experiment-wise $p$ values at $<.05$ (Holland and copenhaver, 1988). A CochranMantel-Haenszel test was employed to test the difference in the unsafe patient handling pre- and postintervention. Descriptive statistics (percentage and frequency) were used to determine the perceived level 
of support for the program and perceived program effectiveness. (See also Table 1, depicting analysis plan for each variable).

\section{Results}

Injury rates: Post-intervention injury rate decreased in 15 of the 23 units, increased in 7 units and remained the same in 1 unit. Overall, the injury rate decreased from 24.0/100 caregivers at baseline and 16.9/100 caregivers post-intervention. Post-intervention injury rates were found to be significantly lower $\chi^{2}(1, n=46)=4.42$, $p=0.036$. Table 2 depicts the injury rates by unit.

Modified duty days: The number of modified duty days decreased significantly $(p=.02)$ from 1,777 modified duty days during the 9 month pre-intervention period, to 539 modified duty days during the 9 month post-intervention period. All but one site saw decreases in the average number of modified duty days taken per injury from pre-to post-intervention (Table 3). The

Table 2

Injury rates by unit (per 100 workers/year)

\begin{tabular}{|c|c|c|c|c|}
\hline \multirow[t]{2}{*}{ Facility } & \multirow[t]{2}{*}{ Unit } & \multicolumn{2}{|l|}{ Injury rates* } & \multirow{2}{*}{$\begin{array}{l}\text { Rate } \\
\text { change }\end{array}$} \\
\hline & & $\begin{array}{l}\text { Pre- } \\
\text { intervention }\end{array}$ & $\begin{array}{l}\text { Post- } \\
\text { intervention }\end{array}$ & \\
\hline \multirow[t]{2}{*}{1} & NHCU-A & 62.2 & 52.7 & $\downarrow 9.5$ \\
\hline & NHCU-B & 23.1 & 33.2 & $\uparrow 10.1$ \\
\hline \multirow[t]{2}{*}{2} & NHCU-A & 14.1 & 3.7 & $\downarrow 10.4$ \\
\hline & NHCU-B & 9.8 & 0 & $\downarrow 9.8$ \\
\hline \multirow[t]{3}{*}{3} & NHCU-A & 13.0 & 8.7 & $\downarrow 4.3$ \\
\hline & NHCU-B & 0 & 0 & 0.0 \\
\hline & SCI & 10.3 & 0 & $\downarrow 10.3$ \\
\hline \multirow[t]{3}{*}{4} & NHCU-A & 23.8 & 21.5 & $\downarrow 2.3$ \\
\hline & NHCU-B & 8.1 & 20.8 & $\uparrow 12.7$ \\
\hline & NHCU-C & 4.1 & 24.7 & $\uparrow 20.6$ \\
\hline \multirow[t]{4}{*}{5} & NHCU-A & 11.2 & 0 & $\downarrow 11.2$ \\
\hline & NHCU-B & 11.9 & 13.1 & $\uparrow 1.2$ \\
\hline & NHCU-C & 4.4 & 0 & $\downarrow 4.4$ \\
\hline & SCI & 0 & 3.7 & $\uparrow 3.7$ \\
\hline \multirow[t]{4}{*}{6} & NHCU-A & 24.1 & 18.1 & $\downarrow 6.0$ \\
\hline & NHCU-B & 19.4 & 22.1 & $\uparrow 2.7$ \\
\hline & NHCU-C & 81.8 & 61.6 & $\downarrow 20.2$ \\
\hline & NHCU-D & 16.2 & 14.4 & $\downarrow 1.8$ \\
\hline \multirow[t]{5}{*}{7} & NHCU-A & 33.0 & 15.8 & $\downarrow 17.2$ \\
\hline & NHCU-B & 41.0 & 13.0 & $\downarrow 28.0$ \\
\hline & NHCU-C & 60.5 & 23.6 & $\downarrow 36.9$ \\
\hline & SCI-A & 21.3 & 4.1 & $\downarrow 17.2$ \\
\hline & SCI-B & 16.3 & 18.8 & $\uparrow 2.5$ \\
\hline Overall & & 24.0 & 16.9 & $\downarrow 7.1$ \\
\hline
\end{tabular}

*Post-intervention injury rates were found to be significantly lower than pre-intervention injury rates based on multiple Poisson regression with $\chi^{2}(1, n=46)=4.42, p=0.036$. median of modified duty days decreased in five sites post-intervention, increased in one site and remained the same in one site. (Table 4). Using the Wilcoxon signedrank test, a statistically significant difference was found between pre-intervention scores for modified duty days $($ median $=10.2)$ and post-intervention $($ median $=6.2)$, $Z(n=22)=-2.3, p=0.02$.

Lost work days: During the 9 month pre-intervention period, 18 caregivers suffered lost work day injuries related to patient handling. These 18 injuries resulted in a total of 256 lost work days, an average of 14.2 days per injury. During the 9-month post-intervention period, 20 caregivers suffered lost workdays, resulting in 209.5 lost workdays, an average of 10.5 days per injury (Table 5). The median of lost work days decreased in five sites post-intervention, and increased in two sites. (Table 6). Wilcoxon signed-rank test was conducted and no statistically significant differences were found for lost days (pre-intervention median $=1$, post-intervention median $=1), Z(n=23)=-0.26$, $p=0.79$.

Self-reported unsafe patient handling and lifting practices: Nurses were asked to self report the number of times in a typical day they performed patient handling tasks unsafely, that is without the proper equipment or number of staff needed. Responses included none, 1-2, $3-4,5-6,7-8,9-10,10+$. There was a statistically significant decrease in the number of self-reported unsafe patient handling practices during the postintervention period based on Cochran-Mantel-Haenzsel $(p=0.027)$. Fig. 2 illustrates self-reported unsafe patient handling acts pre- and post-intervention.

Job satisfaction: Table 7 lists the mean score for each of the six job satisfaction components and the overall job satisfaction score, which was the mean of all survey items. The overall score and five of the six component scores increased from pre-to post-intervention, indicating an increase in job satisfaction (pay, professional status, task requirements, autonomy, and organizational policies). Professional status and task requirements increased significantly.

Perceived support for the program: During preintervention, a total of $675 \mathrm{BIRN}$ perceptions of the level of support from their nurse manager were recorded over 9 months, and $91.5 \%$ of these responses were positive. The percentage increased to $99.4 \%(n=767)$ post-intervention. This indicated that the BIRNS perceived strong support from nurse managers for the program, and that the level of support increased over time. Similarly, BIRNs perceived a strong level of support from their co-workers $(91.1 \%(n=646))$ preintervention, with post-intervention increasing to $97.9 \%$ $(n=764)$. Patients also became more supportive of the program after the interventions were in place. Before the intervention, $71.7 \%(n=512)$ said their patients, residents and/or families were enthusiastic about the 
Table 3

Mean number of modified duty days by site

\begin{tabular}{|c|c|c|c|c|}
\hline \multirow[t]{2}{*}{ Site } & \multicolumn{2}{|l|}{ Pre-intervention } & \multicolumn{2}{|l|}{ Post-intervention } \\
\hline & $\begin{array}{l}\text { \# Injuries with modified } \\
\text { duty days }\end{array}$ & Mean (SD) & $\begin{array}{l}\text { \# Injuries with modified } \\
\text { duty days }\end{array}$ & Mean (SD) \\
\hline 1 & 6 & $50.9(64.3)$ & 7 & $9.6(12.1)$ \\
\hline 2 & 1 & $1.5(0)$ & 3 & $8.0(5.6)$ \\
\hline 3 & 10 & $21.7(16.4)$ & 13 & $8.5(8.2)$ \\
\hline 4 & 18 & $16.4(27.1)$ & 17 & $9.7(10.6)$ \\
\hline 5 & 4 & $10(2.8)$ & 1 & $6(0)$ \\
\hline 6 & 32 & $28.1(33.8)$ & 17 & $9.5(6.6)$ \\
\hline 7 & 3 & $6(3.6)$ & 1 & $5.0(0)$ \\
\hline Total & 74 & $24.0(33.0)$ & 59 & $9.1(8.6)$ \\
\hline
\end{tabular}

Table 4

Median number of modified duty days by site

\begin{tabular}{lll}
\hline Site & $\begin{array}{l}\text { Median number of } \\
\text { modified duty days } \\
\text { by site }\end{array}$ & \\
\cline { 2 - 3 } & Pre-intervention & Post-intervention \\
\hline 1 & 17.8 & 5 \\
2 & 1.5 & 7 \\
3 & 17.0 & 6 \\
4 & 6.5 & 5 \\
5 & 9.0 & 6 \\
6 & 10.0 & 7 \\
7 & 5.0 & 5 \\
\hline
\end{tabular}

program and the percentage increased to $96.1 \%$ $(n=746)$ after the intervention. (Fig. 3)

Perceived effectiveness of program elements: BIRNs on each high risk unit were asked for their perception of the effectiveness of each program element. Post-intervention, the patient handling equipment was ranked \#1, with $96 \%$ of the respondents rating it as "extremely effective". The No lift Policy was ranked as the second highest program element, with $68 \%$ rating it as "extremely effective". Ranked \#3 was the role of the peer safety leader (BIRN), with $66 \%$ rating this program element as "extremely effective". Ranked \#4, 5, and 6 were the Ergonomic Assessment Protocol, Patient Handling Assessment Criteria and Decision Algorithms, and After Action Reviews, which received ratings of $59 \%, 55 \%$, and $41 \%$, respectively (Table 8 ).

\section{Program costs}

Capital costs: A total of $\$ 846,476$ was expended to purchase devices to assist in the handling of patients. These devices included ceiling mounted patient lifts, floor-based full body sling lifts, mechanical lateral transfer aids, powered stand assist lifts, friction-
Table 5

Mean number of lost work days by site

\begin{tabular}{llllll}
\hline Site & \multicolumn{2}{l}{ Pre-intervention } & & \multicolumn{2}{l}{ Post-intervention } \\
\cline { 2 - 3 } \cline { 5 - 6 } & $\begin{array}{l}\text { \# Injuries } \\
\text { with lost } \\
\text { workdays }\end{array}$ & $\begin{array}{l}\text { Mean } \\
\text { (SD) }\end{array}$ & & $\begin{array}{l}\text { \# Injuries } \\
\text { with lost } \\
\text { workdays }\end{array}$ & $\begin{array}{l}\text { Mean } \\
\text { (SD) }\end{array}$ \\
& & & & \\
\hline 1 & 4 & $32.4(28.7)$ & 9 & $9.7(11.3)$ \\
2 & 3 & $4.8(4.5)$ & & $5.0(0)$ \\
3 & 1 & $55.0(0)$ & & $20.2(15.9)$ \\
4 & 1 & $1(0)$ & 1 & $8.0(0)$ \\
5 & 1 & $2(0)$ & 0 & $0(0)$ \\
6 & 7 & $7.4(14.0)$ & 4 & $2.1(2.0)$ \\
7 & 1 & $2(0)$ & 0 & $0(0)$ \\
Total & 18 & $14.2(21.3)$ & 20 & $10.5(12.2)$ \\
\hline
\end{tabular}

Table 6

Median number of lost days by site

\begin{tabular}{lcc}
\hline \multirow{2}{*}{ Site } & \multicolumn{2}{c}{ Median number of lost work days } \\
\cline { 2 - 3 } & Pre-intervention & Post-intervention \\
\hline 1 & 31.8 & 8 \\
2 & 3.0 & 5 \\
3 & 55.0 & 29 \\
4 & 1.0 & 8 \\
5 & 2.0 & 0 \\
6 & 2.0 & 1.5 \\
7 & 2.0 & 0 \\
\hline
\end{tabular}

reducing devices, and gait belts with handles. Capital costs including maintenance and installation totaled $\$ 1,156,266$ with an annualized cost of $\$ 115,627$ for all 23 units combined, based on a 10-year equipment lifeexpectancy. 
Nursing staff training costs: Staff training costs were estimated to be $\$ 74,103$ with an annualized cost of $\$ 7,410$ for all 23 units combined.

Overall program cost: The overall program cost including equipment, maintenance and training over the ten-year period was estimated to be $\$ 123,037$ per year.

\section{Potential cost avoidance}

Cost of medical treatment: The costs associated with workers' compensation, diagnostic tests, and physician services combine to account for the costs of medical treatment. The cost of care billed by facilities to workers' compensation decreased from $\$ 62,702$ at baseline to $\$ 16,260$, post-intervention. The cost of diagnostic tests/medications was also computed. X-rays, Electrocardiograms (EKGs) and computerized axial tomography (CT scans) constituted the major diagnostic tests used for injuries. We computed the average cost, including readings of X-rays as $\$ 95$, EKGs as $\$ 55$ and CAT scans as $\$ 450$. The total of these diagnostic tests remained fairly constant pre- to post-intervention: $\$ 8,610$ compared with $\$ 7,810$. Costs of physician services were estimated based on CPT Codes, and remained fairly consistent: $\$ 23,778$ at baseline and $\$ 25,173$ post-intervention. Combining costs associated

Self-Reported Unsafe Patient Handling ( $n=128)$

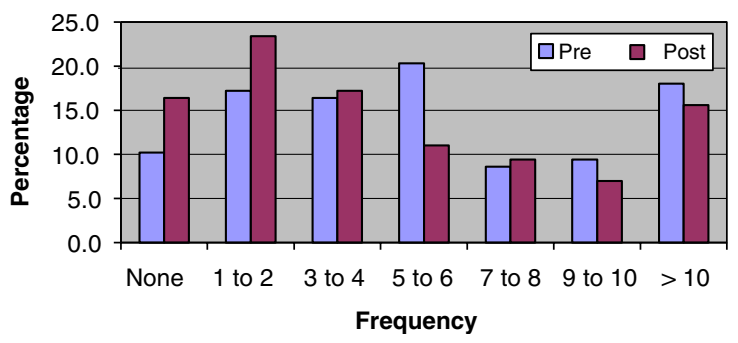

Fig. 2. Self-reported unsafe patient handling post-intervention frequency was found to be significantly lower than preintervention based on Cochran-Mantel-Haenzsel test with $p$ value of 0.027 . with workers' compensation, diagnostic tests, and physician services, the overall total cost of medical treatment decreased from $\$ 95,091$ to $\$ 49,244$.

Workers' compensation costs: Facility Worker's Compensation payments include continuation of payments (COP) to injured personnel when the injury results in up to 45 lost days (short term disability). Beyond 45 days, the Department of Labor provides cash payments for long term disability to individuals who are absent from work. In the pre -intervention stage we estimated COP facility payments for injured employees in the SPHM project at $\$ 134,763$ while for the post-intervention period we estimated that COP payments declined to $\$ 35,200$.

Cost of lost productivity: We characterized lost productivity as the number of personal leave days and lost work days taken for recuperation as a result of a workplace injury (monetary value set equal to the wage rate) and the number of modified duty work days (monetary value set equal to one half the wage rate). The cost of personal days decreased from $\$ 55,743$ to $\$ 49,352$

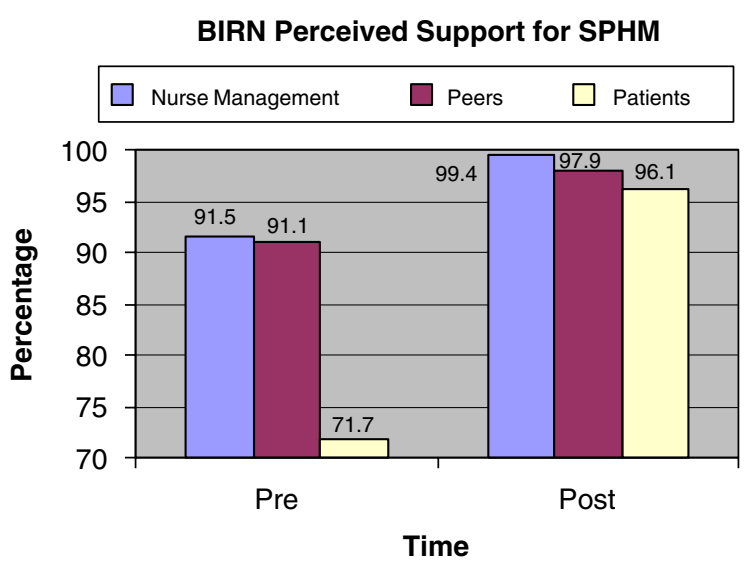

Fig. 3. BIRN perception of program support by nurse management, peers, and patients.

Table 7

Paired $t$-tests between job satisfaction scores

\begin{tabular}{|c|c|c|c|c|c|}
\hline Scores & $N$ & Pre-intervention & Post-intervention & $F$-value & $\operatorname{Pr}>F$ \\
\hline Pay & 145 & 3.42 & 3.54 & 1.65 & 0.20 \\
\hline professional status & 145 & 3.45 & 3.77 & 10.17 & $* 0.001$ \\
\hline Task requirements & 145 & 2.91 & 3.24 & 12.4 & $* 0.0006$ \\
\hline Autonomy & 145 & 3.86 & 4.09 & 4.51 & 0.04 \\
\hline Organizational policies & 145 & 4.03 & 4.13 & 0.98 & 0.32 \\
\hline Interaction & 145 & 3.82 & 3.8 & 0.04 & 0.84 \\
\hline Overall & 145 & 3.63 & 3.79 & 4.5 & 0.04 \\
\hline
\end{tabular}

\footnotetext{
*Experiment-wise $p<0.05$.
} 
Table 8

Perceived effectiveness of program elements pre-intervention and post-intervention

\begin{tabular}{|c|c|c|c|c|c|c|c|}
\hline $\begin{array}{l}\text { Intervention } \\
\text { time period }\end{array}$ & $\begin{array}{l}\text { Not at all } \\
\text { effective }\end{array}$ & $\begin{array}{l}\text { Somewhat } \\
\text { ineffective }\end{array}$ & No effect & $\begin{array}{l}\text { Somewhat } \\
\text { effective }\end{array}$ & $\begin{array}{l}\text { Extremely } \\
\text { effective }\end{array}$ & Unsure & Total \\
\hline \multicolumn{8}{|c|}{ Patient handling and moving equipment } \\
\hline Pre- & $0(0 \%)$ & $2(0.3 \%)$ & $49(6.6 \%)$ & $141(18.9 \%)$ & $547(73.1 \%)$ & $9(1.2 \%)$ & 748 \\
\hline Post- & $2(0.3 \%)$ & $0(0 \%)$ & $1(0.1 \%)$ & $21(2.7 \%)$ & $753(96.4 \%)$ & $4(0.5 \%)$ & 781 \\
\hline \multicolumn{8}{|c|}{ Effectiveness of no lift policy } \\
\hline Pre- & $5(0.7 \%)$ & $6(0.9 \%)$ & $108(15.6 \%)$ & $145(21.0 \%)$ & $261(37.7 \%)$ & $167(24.1 \%)$ & 692 \\
\hline Post- & $0(0 \%)$ & $0(0 \%)$ & $20(2.6 \%)$ & $170(21.9 \%)$ & $530(68.3 \%)$ & $56(7.2 \%)$ & 776 \\
\hline \multicolumn{8}{|c|}{ Effectiveness of BIRN (Back injury resource nurse) } \\
\hline Pre- & $2(0.3 \%)$ & $4(0.5 \%)$ & $42(5.6 \%)$ & $347(46 \%)$ & $333(44.2 \%)$ & $26(3.4 \%)$ & 754 \\
\hline Post- & $0(0 \%)$ & $5(0.6 \%)$ & $38(4.9 \%)$ & $216(27.7 \%)$ & $518(66.3 \%)$ & $4(0.5 \%)$ & 781 \\
\hline \multicolumn{8}{|c|}{ Effectiveness of ergonomic assessment protocol } \\
\hline Pre- & $4(0.6 \%)$ & $5(0.7 \%)$ & $148(21.5 \%)$ & $160(23.2 \%)$ & $135(19.6 \%)$ & $237(34.4 \%)$ & 689 \\
\hline Post- & $3(0.4 \%)$ & $0(0 \%)$ & $54(6.9 \%)$ & $199(25.3 \%)$ & $461(58.6 \%)$ & $70(8.9 \%)$ & 787 \\
\hline \multicolumn{8}{|c|}{ Effectiveness of patient handling assessment criteria and decision algorithms } \\
\hline Pre- & $9(1.5 \%)$ & $2(0.3 \%)$ & $146(23.6 \%)$ & $119(19.3 \%)$ & $103(16.7 \%)$ & $239(38.7 \%)$ & 618 \\
\hline Post- & $19(2.5 \%)$ & $0(0 \%)$ & $37(4.9 \%)$ & $235(31.0 \%)$ & $417(54.9 \%)$ & $51(6.7 \%)$ & 759 \\
\hline \multicolumn{8}{|c|}{ Effectiveness of AAR (after action review) } \\
\hline Pre- & $8(1.1 \%)$ & $5(0.7 \%)$ & $175(25.1 \%)$ & $181(26.0 \%)$ & $165(23.7 \%)$ & $162(23.3 \%)$ & 696 \\
\hline Post- & $10(1.3 \%)$ & $3(0.4 \%)$ & $164(21.7 \%)$ & $161(21.3 \%)$ & $308(40.8 \%)$ & $109(14.4 \%)$ & 755 \\
\hline
\end{tabular}

and the cost of modified days decreased from $\$ 136,426$ to $\$ 42,500$.

Overall injury costs: Cost of medical treatment, Workers' Comp costs, and costs of lost work days, personal leave days and modified duty days were totaled to obtain injury cost values for pre -and post-intervention, with a savings of $\$ 245,727$.

Expected cost savings: The annualized expected overall injury costs savings was calculated to be $\$ 327,636$. Taking out the program costs per year of $\$ 123,037$, the overall annualized cost savings per year was $\$ 204,599$.

Cost/benefit summary: Over a 10-year period, this $\$ 204,599$ cost savings per year translates to over $\$ 2$ million dollars of savings, excluding the effect of inflation. The initial capital investment for patient handling equipment and training was recovered in approximately 3.75 years based on annual post-intervention savings in workers' compensation expenses and costs associated with lost and modified work days.

Rate of return on investment: The higher the IRR, the more attractive it is as a financial investment. Computations yielded an IRR of close to $19 \%$ for the safe patient handling and movement project, indicating a very high return on investment.

Indirect costs not included in the analysis: The indirect costs of injury and low morale among workers has been well documented in the literature. A high incidence of injury undoubtedly results in increased caregiver exodus costing the facilities in terms of lost productivity and training costs. We did not include these intangible costs in our analysis because of the subjective nature of assigning monetary values to these costs. Such indirect costs have been estimated as high as five times the direct cost though an estimate of twice the direct costs is an accepted measure. Savings in costs related to recruitment and retention of nurses can be substantial in a workforce traditionally plagued by shortages. Hence, we feel this study conservatively underestimates the social value of the interventions implemented.

\section{Discussion}

Nurses have one of the highest incidences of musculoskeletal work related back injuries of any profession. Over the past 30 years, efforts to reduce work-related musculoskeletal disorders in nurses have been largely unsuccessful. This study tested a newly developed patient care ergonomics program designed to create safer working environments for nurses who provide direct patient care.

This study is consistent with other field studies supporting the use of patient handling equipment as part of a comprehensive patient care ergonomics program to reduce work-related musculoskeletal injuries in careivers. Collins et al., (2004) implemented a similar multi-faceted program including patient handling equipment, comprehensive training on proper lift use, and a 
zero lift policy that resulted in significant decreases in injury rate and lost workday and restricted workday injury rates. Costs of injuries to caregivers associated with lifting and moving residents also decreased. Similarly, after deployment of mechanical patient lifts, another team of investigators reported reductions in injury rates, lost workday injury rates, workers' compensation costs, and musculoskeletal symptoms (Li et al., 2004).

Our program resulted in improvements in injury rates, modified duty days, job satisfaction, costs, and selfreported performance of 'unsafe' patient handling practices, however, while the number of lost work days decreased, this change was not statistically significant. Further, while injury rates decreased in 15 of the 23 units, 7 units actually reported a slight increase in injury rates and 1 unit remained unchanged.

The complex, contextual aspect of the work environment cannot be ignored, and these issues may help explain results from this study, as well as finding from other multifaceted intervention programs designed to reduce risk to caregivers. Each of these issues will be briefly discussed.

First, nurses notoriously under report injuries (Yassi et al., 1985; Collins et al., 2004). A national survey was conducted in Scotland (McGuire and Dewar, 1995), which included 5,184 randomly selected nurses $(73 \%$ response rate $N=4918$ ). One area examined was reporting of injuries. $33.4 \%$ of subjects had sustained an injury related to patient handling but only $52 \%$ of those with an injury had reported it. Several reasons for under reporting have been documented in the literature: (1) unawareness of injury until after the event (waking up sore, calling in, and deciding after the fact to ignore it) (McGuire and Dewar, 1995; Retsas and Pinikahana, 2000), (2) confusion over whether going home with a dull ache is considered an injury (Collins, 1990; McGuire and Dewar, 1995), (3) bureaucracy associated with reporting an injury is determined to be too great a barrier, particularly when the injury is minor and /or not physically debilitating (Collins, 1990; James, 1989; McGuire and Dewar, 1995; Retsas and Pinikahana, 2000); (4) fear of dismissal (Collins, 1990; Retsas and Pinikahana, 2000) or of being stigmatized (Collins, 1990; Retsas and Pinikahana, 2000), (5) socialization of nurses to focus on patient care and deny their own needs/safety (Collins, 1990), (6) peer pressure not to take time off for injury, because rarely are caregivers replaced on the job; taking time off increases the exposure of the nurses left at work, who also have pain and may be less than supportive of an injured worker (Collins, 1990), and (7) perceived lack of support from occupational health providers who may be dismissive of reports of back pain, discouraging reporting, particularly since much of the medical evaluation is subjective and there are not clear diagnostic tests (Collins, 1990). Under reporting of occupational injuries in nursing affects the evaluation of interventions. Baseline injury data are much lower than expected, while efforts to raise the awareness of safety and occupational injuries can lead to an increase in reporting post-intervention. With a programmatic emphasis on staff education and a culture of safety, it is possible to heighten the awareness and increase reporting. While our study showed a significant decrease in injuries from pre-to post-intervention, the magnitude of this difference may be hidden. In three of our study units where an increase in lost work days was reported postintervention, focus groups held with staff at baseline revealed a fear of reporting injuries, based either on retribution expected from peers or superiors. In these areas, an increase in reporting could actually be viewed as a positive outcome, supporting a healthier culture of safety.

Another factor is the issue of specifying endpoints for injury data. As is common in studies capturing injury data from cumulative injuries, problems with the endpoints for injury data were noted for both lost workdays and modified workdays. For example, a lost workday injury during pre-intervention time period can carry over into post-intervention time period, just as a lost workday in post-intervention may continue after the study is concluded. This inability to follow all lost time and modified duty days to closure may result in excluding important injury information. Most importantly, since the link between exposure and injury has not yet been established for patient handling injuries, the onset of injury in post-intervention may have been related to exposure that occurred pre-intervention. Another challenge is the management of multiple injuries within the same subject, as well as re-injury that may or may not be job-related.

Nursing turnover rates also impede data collection and skew results. For example, it is difficult to determine the denominator when calculating injury rates, since the number of staff on a unit varies significantly over time combined with the difficulty of capturing staff that 'float' into and out of a unit on a shift-by-shift basis. Also, on units with high turnover rates, injured staff may leave the participating unit, hampering follow-up efforts for both baseline and post-intervention periods. The turnover of nursing staff on some of the units in this study exceeded $65 \%$. This means that staff cohorts pre and post intervention were largely different. Further, since injuries tend to be cumulative, we were not able to clearly estimate exposure in new staff, who worked in other settings.

Our study follow up period was 9 months, a timeframe adequate to address immediate impacts of the program, but not likely to fully evaluate the impact of the program longitudinally. A minimum of 2-3 years of post-intervention data collection is typically necessary to give adequate time for capturing improvements, 
especially related to musculoskeletal discomfort outcome measures (Collins, 1990). It is likely that the short post-intervention data collection period of our study was insufficient to detect longer term benefits related to the decreasing severity of injuries.

Another issue likely to affect our study is the limitations of self-report data. Injuries were selfreported and it is possible that the data associations may have been associated with common method variance (Spector, 1994), hypochondria (Ferguson, 1998) and/or negative affectivity (Watson and Clark, 1984). But, because physical examination may not be able to detect physical injury when non-specific pain is reported (Griffiths, 1994; Greening and Lynn, 1998), self-report may be the only way to capture this information. Randall and colleagues (2002) noted that the challenge is to develop reliable measures and to understand and make suitable allowance for sources of bias in such reports. Given musculoskeletal damage, which can only be detected using the most sensitive of measuring devices, may be associated with real discomfort (Greening and Lynn, 1998), such data may also be the only practical way of identifying the early stages of the development of musculoskeletal problems. For other self-reported data we used strategies such as triangulation, whereby we obtained perceptions from multiple sources as a way to validate findings.

Additionally, there are confounding variables likely to affect our results. Work related exposure is difficult to capture given staff turnover, frequent floating to other units, and exposure in previous jobs. Further, the etiology of cumulative injuries is not always clear. Consider, for example, a nurse who is working two jobs, or who participates in non-work related activities likely to contribute to a cumulative injury. Further, a nurse who is detailed to another unit where they lack proper patient equipment and a no lift policy may be injured, and this injury is recorded on the original unit for which they were assigned. Key confounders not controlled for in this study include social aspects of the work and workplace; physical and social aspects of life outside the workplace; economic incentives/cultural values; and physical/psychological characteristics of the individual (Panel on Musculoskeletal Disorders and the Workplace, 2001). We did, however, control for type of nursing units, level of nursing personnel, type of equipment available, and other aspects of the organization in the work environment. Further, we triangulated data from multiple sources, e.g., BIRN reports, focus groups of nursing staff, focus groups with managers, patient interviews, surveys, and injury reports.

Other temporal issues include the difficulty of having precise pre- and post-intervention data collection time periods. With the necessary phasing in of program elements and patient handling equipment, and the need for time for staff to adapt to new equipment and procedures, it is difficult to have a precise start point for post-intervention. To counter this, we allowed a onemonth settling-in period between phasing in of program elements and equipment and post-intervention data collection but this time period was insufficient. We recommend extending that time period to a minimum of two to three months.

Program fidelity is a critical issue. When designing multifaceted programs, you cannot tell which program elements most contributed to the success/non-success. Further, as with any multi-site intervention study the dose of the intervention varies by site and over time. We maintained close contact with each site and maintained monthly process logs to assure that an adequate dose of the intervention was implemented and maintained over time.

\section{Conclusions and recommendations for future research}

This multi-faceted program resulted in positive outcomes associated with injury rates, modified duty days, job satisfaction, self-reported safety in performing patient handling tasks, and cost. The program was well accepted by patients, nursing staff, and administrators. While the total number of lost workdays decreased by $18 \%$ post-intervention, this difference was not statistically significant. We believe the program was successful in the short term. Further research is needed to evaluate long term impact of the program. While the program was deemed effective, there is still significant room for improvement in creating safe work environments for nursing staff.

Our multifaceted program focused interventions on high-risk units, where there is significant exposure to "at risk" tasks, such as patient lifts and transfers. This target group offered the most opportunity for improvement. Future research is needed to develop and test multifaceted programs for moderate risk units or areas with acute nursing shortages, such as critical care, operating room, and medical/surgical units.

This study showed that the reduction in workers' compensation medical and indemnity expenses effectively recovered the initial capital investment in equipment and training costs in approximately 3.75 years and potentially more quickly if indirect cost savings are considered. Further research is needed to justify capital investment in patient handling equipment and other program elements, focusing on program costs, return on investment, and cost effectiveness. This financial justification is a critical step for administrators consider whether to allocate resources to a patient care ergonomics program. Efforts to build a business case for patient care ergonomics would facilitate implementation of this program on a national level. 
Under reporting of patient handling injuries is a critical problem (US Department of Health Human Services (USDHHS), 1999), and consequently is a major obstacle to studying nursing injuries Further research is needed to examine how nursing staff make decisions about when to report chronic musculoskeletal pain as an "injury". Further complicating reporting, injury report forms are often designed to capture acute injuries and may fail to capture important information about cumulative injuries. Injury forms need to be revised to more fully describe cumulative injuries in a way that is not burdensome the nurse reporting the injury. Lastly, the culture of injury reporting on units influences the number of injuries reported.

Because musculoskeletal pain and discomfort are considered the initial symptoms of musculoskeletal impairment and disability (US Department of Health Human Services (USDHHS), 1999) and most patient handling injuries are cumulative in nature, further research is needed to physiologically characterize musculoskeletal cumulative trauma injuries to determine at what point the threshold of a muscle for micro-tears is exceeded and an acute injury develops. Randall et al., (2002) and Menzel et al., (2004) corroborated the need to study musculoskeletal discomfort more completely to become aware of the nuances of musculoskeletal pain.

Technology holds much promise as a solution for reducing risks associated with patient handling, but care needs to be taken to include direct care providers in equipment purchase decisions. Also, few facilities have developed equipment maintenance and repair programs. Lack of such programs interferes with safe and optimal use of equipment.

While the patient handling equipment was well accepted by staff and patients, and rated as the most effective aspect of the program by caregivers, gaps in technology were noted. Currently, there are no viable technology solutions for a high-risk, high-volume patient handling task: repositioning a patient in a bed or chair. Anecdotal data from our study revealed postintervention, musculoskeletal discomfort was associated with repositioning tasks. Further development research is needed to design prototypes for managing this key patient handling task.

Lastly, while this study focused on caregiver safety, issues related to patient safety and quality of care need further study. Nurses with chronic musculoskeletal pain are less likely to move physically dependent patients or provide high-risks tasks that would jeopardize their health. Dedicated nursing staff who force themselves to provide these tasks while in pain, could inadvertently drop a patient or cause skin tears while dragging a patient across surfaces during a transfer. On units where staffing is short, injured nurses working on modified duty status further tax the staffing resources and increase the exposure of their coworker's to a higher concentration of high risks tasks. More research is needed on the role staffing (and indirectly, risk exposure) in patient care related injuries.

This study has several limitations. First, there was no control group. Secondly, the data collection interval was brief ( 9 months), which did not allow for long term benefits to be identified. Given the cumulative nature of nursing injuries, a 3 year follow up period would be ideal. Also, some bias may have been introduced by asking respondents to self-report outcome measures.

Lessons learned include:

- Fiscal outlay for patient-handling equipment may initially appear to be prohibitive, but the program is cost-effective. The highest cost item was the patient handling equipment, which was perceived by nurses as the most beneficial program element. In addition, there are ways to work around initial equipment expense by working with manufacturers or workers compensation insurance carriers, or using creative financing or acquisition methods.

- Patients are less likely than nursing staff and administrators to embrace new patient handling technologies and practices at the onset of the program, however, over time, patients and their family members grow to accept these innovations.

- The role of the back injury resource nurse (BIRN) offers an opportunity to empower nurses to modify their work environment to promote safety. This peer leader program is much more effective than traditional educational approaches, and has the added benefit of facilitating implementation of the program as well as to help sustain the program over time.

- Nurses need additional training to fully participate in ergonomic assessments of work environments. However, the unique work environment of nurses, combined with high levels of risk and environmental hazards, warrants closer collaboration between ergonomists and direct patient care providers.

\section{Acknowledgement}

The authors would like to thank James W. Collins, Ph.D., MSME, Associate Director of Science, Division of Safety Research at the National Institute for Occupational Safety and Health (NIOSH) and Bernice Owen, Ph.D., RN for their thoughtful critique of this manuscript.

\section{Disclaimer}

The research reported here was supported by the Department of Veterans Affairs, Veterans Health Administration (VHA), Health Services Research \& Development and New Program Initiatives. The views 
expressed in this article are those of the authors and do not necessarily represent the views of the Department of Veterans Affairs.

\section{References}

Anderson, J., 1980. Back pain and occupation. In: Jayson, M.I.V. (Ed.), The Lumbar Spine and Back Pain, 2nd ed. Pitman Medical Ltd., London, pp. 57-82.

Brown, J., 1972. Manual lifting and related fields. An Annotated Bibliography. Labor Safety Council of Ontario, Ontario, Canada.

Buckle, P., 1981. A multidisciplinary investigation of factors associated with low back pain. Ph.D. Thesis, Cranfield Institute of Technology.

Buckle, P., 1987. Epidemiological aspects of back pain within the nursing profession. International Journal of Nursing Studies 24 (4), 319-324.

Bureau of Labor Statistics (BLS) 2002. Survey of occupational inquiries and illnesses, 2001. US Department of Labor. USDL December 19, 2002.

Bureau of Labor Statistics (BLS) 2003. Highest Incidencd Rates of Total Nonfatal Occupational Injury and Illness Cases, Private Industry, 2002. Author, Washington, DC.

Collins, M., 1990. Occupational Back Pain in Nursing: Development, Implementation and Evaluation of a Comprehensive Prevention Program. Worksafe Australia, Australia, National Occupational Health and Safety Commission.

Collins, J.W., Wolf, L., Bell, J., Evanoff, B., 2004. An evaluatio of a "best practices" musculoskeletal injury prevention program in nursing homes. Injury Prevention 10, 206-211.

Daltroy, L., 1997. A controlled trial of an educational program to prevent low back injuries. The New England Journal of Medicine 337, 322-328.

Daws, J., 1981. Lifting and moving patients, 3, A revision training programme. Nursing Times 77 (48), 2067-2069.

Dehlin, O., Hedenrud, B., Horal, J., 1976. Back symptoms in nursing assistants in a geriatric hospital. Scandinavian Journal of Rehabilitation Medicine 8 (2), 47-53.

Ferguson, E., 1998. Hypochondriacal concerns, symptom reporting and secondary gain mechanisms. British Journal of Medical Psychology 71, 281-295.

Fragala, G., Bailey, L.P., 2003. Addressing occupational strains and sprains: musculoskeletal injuries in hospitals. AAOHN 51 (6), 252-259.

Greening, J., Lynn, B., 1998. Vibration sense in the upper limb in patients with repetitive strain injury and a group of atrisk office workers. International Archives Occupational and Environmental Health 71 (1), 29-34.

Griffiths, A., 1994. Musculoskeletal upper-limb disorders and white-collar work in the UK. Work and Stress 8, 1-3.

Harber, P., Pena, L., Hsu, P., Billet, E., Greer, D., Kim, K., 1994. Personal history, training and worksite as predictors of back pain of nurses. American Journal of Industrial Medicine 25 (4), 519-526.

Hayne, C., 1984. Ergonomics and back pain. Physiotherapy 70 (1), 9-13.

Hignett, S., 1996. Work-related back pain in nurses. Journal of Advanced Nursing 23 (6), 1238-1246.
Hignett, S. 2003. Intervention strategies to reduce musculoskeletal injuries associated with handling patients: a systematic review. Occupational Environmental Medicine 60(e6). Available at: <http://www.occenvmed.com/chi/content/ full/60/9/e6>.

Hodgson, M. 2001. Personal communication.

Holland, B.S., Copenhaver, M.D., 1988. Improved Bonferonitype multiple testing procedures. Psychological Bulletin 104, $145-159$.

James, C., 1989. Social sequelae of occupational injury and illness. Unpublished Ph.D. Thesis, Griffith University, June 1989.

Lagerstrom, M., Hagberg, M., 1997. Evaluation of a 3 year education and training program for nursing personnel at a Swedish hospital. AAOHN Journal 45 (2), 83-92.

Lahad, A., Malter, A.D., Berg, A.O., Deyo, R.A., 1994. The effectiveness of four interventions for the prevention of low back pain. Journal of American Medical Association 272 (16), 1286-1291.

Larese, F., Fiorito, A., 1994. Musculoskeletal disorders in hospital nurses: a comparison between two hospitals. Ergonomics 37 (7), 1205-1211.

Li, J., Wolf, L., Evanoff, B., 2004. Use of mechanical patient lifts decreased musculoskeletal symptoms and injuries among health care workers. Injury Prevention 10 (4), 212-216.

McGuire, T., Dewar, J., 1995. An assessment of moving and handling practices among Scottish nurses. Nursing Standard 9 (40), 35-39.

Menzel, N.N., Brooks, S.M., Bernard, T.E., Nelson, A., 2004. The physical workload of nursing personnel: association with musculoskeletal discomfort. International Journal of Nursing Studies 41 (8), 859-867.

Nelson, A. (Ed.), 2003. Patient Care Ergonomics Resource Guide: Safe Patient Handling and Movement. Tampa, FL: Veterans Administration VISN 8 Patient Safety Center of Inquiry. Available at: http://www.patientsafetycenter.com.

Nelson, A.L., Fragala, G., Menzel, N., 2003a. Myths and facts about back injuries in nursing. American Journal of Nursing 103 (2), 32-40.

Nelson, A.L., Owen, B., Lloyd, J., Fragala, G., Matz, M., Amato, M., Bowers, J., Moss-Cureton, S., Ramsey, G., Lentz, K., 2003b. Safe patient handling \& movement. American Journal of Nursing 103 (3), 32-43.

Nelson, A.L., Powell-Cope, G., Gavin-Dreschnack, D., Quigley, P., Bulat, T., Baptiste, A., Applegarth, S., Friedman, Y., 2004. Technology to promote safe mobility in elderly. Nursing Clinics of North America 39 (3), 649-671.

Panel on Musculoskeletal Disorders and the Workplace; Commission on Behavioral and Social Sciences and Education; National Research Council; and Institute of Medicine, 2001. Musculoskeletal disorders and the workplace: low Back and Upper Extremities. National Academy Press, Washington, DC.

Randall, R., Griffiths, A., Cox, T., Welsh, C., 2002. The activation of mechanisms linking judgments of work design and management with musculoskeletal pain. Ergonomics 45 (1), 13-31.

Retsas, A., Pinikahana, J., 2000. Manual handling activities and injuries among nurses: An Australian hospital study. Journal of Advanced Nursing 31 (4), 875-883. 
Shaw, R., 1981. Creating back care awareness. Dimensions in Health Service 58 (3), 32-33.

Snook, S., Campanelli, R., Hart, J., 1978. A study of three preventative approaches to low back injury. Journal of Occupational Medicine 20 (7), 478-481.

Spector, P.E., 1994. Using self-report measures in organizational behavior research: a comment on the use of controversial method. Journal of Organizational Behavior 15, 385-392.

St. Vincent, M., Tellier, C., Lortie, M., 1989. Training in handling: an evaluative study. Ergonomics 32 (2), 191-210.

Stamps, P., 1997. Nurses and Work Satisfaction: An Index for Measurement, 2nd ed. Health Administration Press, Chicago.

Stetler, C.B., Burns, M., Sander-Buscemi, K., Morsi, D., Grunwald, E., 2003. Use of evidence for prevention of work-related musculoskeletal injuries. Orthopaedic Nursing 22 (1), 32-41.
Stubbs, D., Buckle, P., Hudson, M., Rivers, P., 1983. Back pain in the nursing profession II. The effectiveness of training. Ergonomics 26 (8), 767-779.

US Department of Health and Human Services (USDHHS), 1999. Federal Register, Part II, Department of Labor, Occupational Safety and Health Administration. 29 CFR Part 1910; Ergonomics Program: Proposal Rule (Tuesday, 23 November 1999).

Venning, P., 1988. Back injury prevention among nursing personnel. AAOHN Journal 36 (8), 327-333.

Watson, D., Clark, L.A., 1984. Negative affectivity: the disposition to experience aversive emotional states. Psychological Bulletin 96 (3), 465-490.

Yassi, A., Tate, R., Cooper, JE., Snow, C., Vallentyne, S., Khokhar, J., 1985. Early intervention for back-injured nurses at a large Canadian tertiary care hospital: an evaluation of the effectiveness and cost benefit of a 2-year pilot project. Occupational. Medicine, 45 (4), 209-214. 